\title{
Research on the Development Trend of Modern Sports Teaching Mode from the Perspective of Comprehensive Physical Ascension
}

\author{
Wang Yong \\ Dalian Maritime University, No.1 Linghai Road, \\ Dalian, Liaoning Province, 116085 China
}

\begin{abstract}
In this paper, we conduct research on the development trend of the modern sports teaching mode from perspective of comprehensive physical ascension. Sports teaching is basic organization form of college sports, is the main way to attain the goal of college sports. Colleges and universities sports teaching not only should pay attention to students during the period of school physical exercise and its effect, more important is to teach students how to carry out lifelong physical exercise. To make the students out of school after, still can effectively exercise according to actual condition, so as to make it for life. At home about the definition of teaching model, the majority of acceptance is defined as the teaching mode in a certain teaching idea or theory guidance for the design and organization of teaching in practice, set up the basic structure of the various types of teaching activities and activity program and its implementation method of the strategy system. Our research suggests our proposed new approach of education based on the background that is innovative.
\end{abstract}

Keywords- Sports Teaching, Comprehensive Physical, Development Trend, Ascension.

\section{Introduction}

College campus sports culture refers to the college campus within a specific space environment, and presents a kind of college students as the main body, dominated by college teachers, as the main content on a variety of sports activities, campus spirit as the main features, has a unique form of a kind of group culture that is the interaction between teachers and students in sports activities to create the cultural atmosphere. With the large-scale enrollment expansion of colleges and universities, students' comprehensive quality in overall, combined with the degree of the difference, a lot of the culture of college students could not reach the requirements of modern society for college students. Led directly to the college students' awareness of basic sports culture and sports culture in universities there is a deviation and not value, some even have one-sided understanding so culture is one of the important asymmetry factors of university sports culture construction [1-3].

According to the literature survey, the primary challenges of the physical education could be listed as the follows. (1) At present, our country's colleges and universities sports teaching materials are mostly around teaching sports skills, general writing, machine-made, perennial unchanged, teaching content of sports teaching did not reflect the current social development the content of the training needs that do not tally with the age, poor practicability. In addition, the sports teaching material arrangement most is given priority to with sports individual teaching and training, from the training goal of modern sports teaching, to some extent, ignored the majority of the students participate in demand. (2) Cultivate the students' lifelong sports consciousness, make its master one or two kinds of sports skills and lifelong exercise methods, is the realization of China's higher sports education goal value orientation from social values into personal value, 
diversification towards effective approach of cultivating the ability of independent movement. (3) This kind of fixed mode, while reached a certain amount of exercise effect, but can bring negative impact of boring to students, make them gradually lose interest in physical education. This teaching process too much emphasis on the implementation of classroom teaching mode is a teacher of the subjective structure model. This pattern dominated by teachers which give the priority to with simple teaching skills, ignore students' physical and mental development. Although looks unified teaching process and the specification, but the lack of students' personality and special skills of pertinence, students get the emotional experience by physical exercise and are difficult to implement the needs of the pleasure of body and mind [4-5].
To examine from the angle of teaching ontology teacher main body and the relationship between the teaching object, teaching activity main points to the teachers, organization, and the selection of the curriculum, teaching material processing and evaluation activities; The selection of teaching means, improvement and innovation; The layout of space equipment, the use of sports facilities, etc. From students, on the other hand, the relationship between the subject and the teaching material object to analyze the teaching activities mainly include the students understanding of the teaching materials, curriculum, application and evaluation, the understanding of specific teaching means, methods, and adopt and feedback; The understanding of sports and the practice, etc.

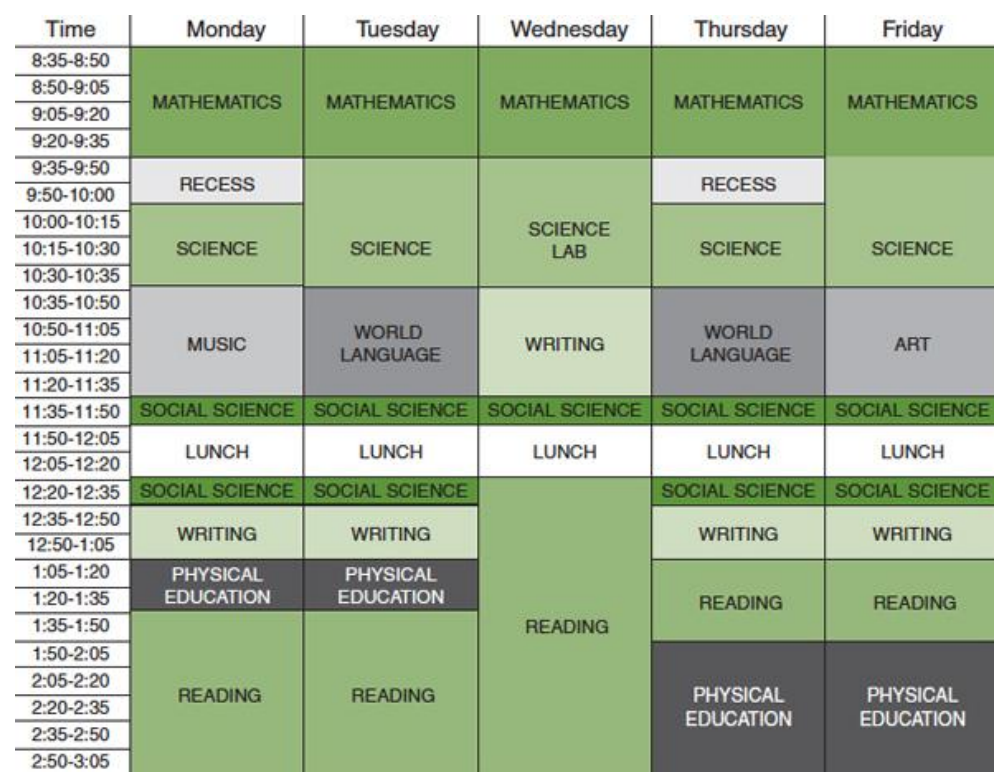

Figure 1. The Sample Schedule of the Modern Physical Education

In this paper, we conduct research on the development trend of modern sports teaching mode from the perspective of comprehensive physical ascension. Schools to the general transformation of higher vocational colleges, which not only provides more teenagers with the opportunity of higher education, also for our country's population quality and the improvement of population quality set up a new learning platform. Higher vocational colleges and universities sports education as an important part of higher vocational education, how to abandon the old idea and the mode of the original medium sports education, create new sports teaching system accords with the feature of higher vocational education, is suspends in front of our physical education workers is an important new topic. 


\section{The Proposed Methodology}

The Comprehensive Physical Ascension. Physical training has become a hot competitive sports training and scientific research at home and abroad, physical fitness is the extrinsic manifestation of sports skill, athletic ability is an important part of. Scientific and reasonable physical training can help the athletes to promote special need to sport ability, improve the body function, improve body shape, and make it more adaptable to the demand of the fierce competition, also can promote the recovery of injury prevention and fatigue and improve athlete special performance [6].

Physical training has experienced the development course of the one hundred, today also go to the science training theory it also requires us to explore the basic structure of physical training. Construct the training structure to consider many factors, such as the conditions of the training, the training of the auxiliary means, supportive training means as auxiliary facilities, the pre-match training and the master, and so on. Training structure in everyone's understanding and the constantly improvement in practice. Their awareness training structure, can only reach a limited depth, in this limited depth, there is only the temporary scenario, this for the ultimate goal, but is a defect that are worth noting. In the training work, we have to learn new knowledge, more carries on the practice, so as to improve our deep understanding of training structure [7].

According to the energy source and that can be put it into genetic fitness and training of physical fitness, people's physical condition is not only with related training methods, training environment and training level is closely related to genetic factors. Need to point out that physical training feature is not only people trained acquired after birth, and use a variety of means when unborn or environment with the purpose of the fetus, consciously get without exercise. In the above several understanding of physical concepts, there are aspects from the access, physical fitness is considered by congenital and acquired training means from the aspects of physiological basis think that physical fitness is a body in morphological structure, physiological function and sports quality aspect of comprehensive sport ability. Different perspective in the study of sports, due to the task and purpose is different that is the concept of fitness, research from different angles need to define also differ.

Training methods and means of design should not only according to the basic characteristics of the project, technical characteristics and the individual differences, but also attaches great importance to improve the function of different degree of the balance of muscle strength, pay attention to improve the large muscle groups and the degree of equilibrium between the small muscles, pay attention to the ability to improve proprioception, pay attention to the weak part of improving the capability of the different athletes, at the same time to improve the special athletes different body chain each link the best proportion of power. Timely recovery of fatigue is an important factor affecting the quality of the training, especially nerve for muscle control ability demanding project is more important. Therefore, scientific training process monitoring, timely adjust the training load, avoid the negative effect of the training methods and means as can promote the effective recovery of fatigue, thus improve the effect of overall physical training paradigm and pattern.

The Physical Enhancement. Physique is the person's morphology, have the ability to change it through the day after tomorrow's sports; Physical fitness is refers to the basic human body each organ system function and muscle activities and behave function ability can also through scientific exercise to improve it the day after the tomorrow. Development and improve the physical process can cause a series of the morphological structure and physiological function changes, and with the change of morphological structure physiological kinetic energy and the improvement of physical fitness, 
and will produce certain psychological process and individual mental characteristics, so as to promote the development of people's mental health.

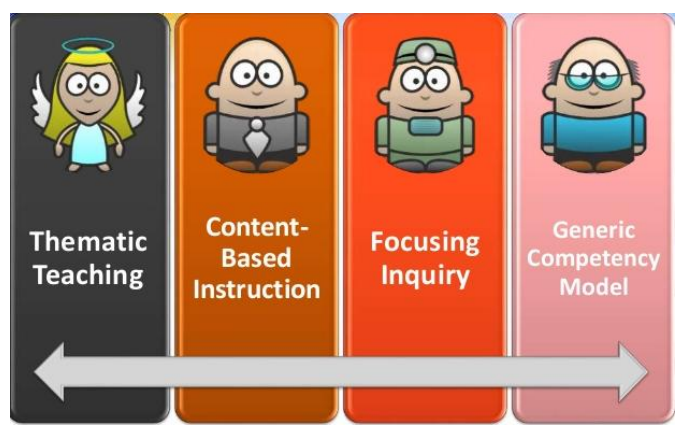

Figure 2. The Sample Instruction Procedures an Steps

Student physical fitness is the students' ability to adapt to the study and life of the body function, sport ability and the objective reflection of life adaptation ability to fight disease. Students to develop the physical the purpose is to promote health, not athletic. Therefore, to strengthen college students' physical fitness should be the focus of the development of students' basic physical fitness factors that affect students' physical fitness that the genetic, exercise, psychological, environmental, lifestyle, etc. However, the main influence factor is the lack of physical exercise, and there are many reasons for the lack of physical exercise. Based on the literature review, we propose the suggestions for the modern physical enhancement pattern as follows. (1) Strengthen aerobic training, enhance cardiopulmonary function. Aerobic training as is one of the basic practice in the directional movement special physical ability, good cardiopulmonary function, the side reflects the ability of carrying oxygen of blood in the body is good, can adapt to the terrain, weather, the match in match distance, exercise intensity and various uncertainty factors bring a variety of adverse reactions that avoid physical consumption too rapidly which also provide the favorable conditions for the competition. (2) Understand each player's physical characteristics, targeted to arrange the training task. Directional movement of the training is divided into two large pieces of special physical fitness and special technology, whether that piece of training, should according to their aptitude and treated differently, in order to achieve the desired effect of the finite time effective training. (3) Strengthen the body foundation quality is fundamental. Understands from actual condition, the tested players are cultural achievement are relatively good, the directional movement is most own hobby, and amateur athletes. Based on students' learning pressure big, participate in the directional movement training time is very limited, for this reason, we must start from foundation quality consolidate the team on the general basis of quality and gradually increase the quality of the special physical ability training [8].

The Physical Education Optimization. College sports teaching reform that must pay attention to training on college students' lifelong health idea, on the contents of physical education curriculum and teaching material, to lifelong fitness as the main content. Attaches great importance to the national fitness education, train students' correct learning motivation, as well as strengthen students' physique, strengthen the learning of lifetime sports theory guidance and technical skills, get into the habit of regular exercise the body, set up the lifelong sports concept neither from the actual physical education teaching reform, and also not from the era and the development of the society, but in the gradual scientific orbit. The problem of the relationship between the teaching and learning that is the starting point of the formation and development of teaching theory which is composed of basic elements in understanding and established. The research has been an important subject in the study of teaching theory. Teaching is the teacher through a series of logical thinking process of cognition, judgment and reasoning, and obtains the objective of teaching practice rationality. Teaching practice no matter when and where is a concept as the guide. Say, 
how many kinds of understanding on the relationship between the teaching and learning, there will be many different kinds of the teaching theory system. Because people have different understanding of the problem of the relationship between teaching and learning, understanding of the concept of "teaching" are different.

Teaching process has certain default, but it is essentially a generated, namely, teaching activities is uncertain, not causality, unpredictability and the generative nature. Because of the specific teaching activities is no fixed pattern to be found that is not repeatable, a variety of unexpected event in the teaching is inevitable. PE teaching activities, teaching environment outdoor in the changing dynamic every time, even if the students of the same age level due to the different physical condition, physical quality, such as learning basis, also at the scene of very significant differences on motor skill learning, so as an experienced teacher can't only stay in the textbooks, and class teaching design, more in need of changing teaching practice according to their aptitude. Of the study of physical education teaching reform in colleges and universities, the main attention to the student personality cultivation and the potential development, the center of gravity, insisted that take the student as the main body, respect students individual difference, strive to make every student to get succeed. Second is to reform the existing sports teaching method, to break the traditional centralized teaching mode, bold practice the open and exploratory teaching method, etc. After the reforms of the recent years, the sports teaching achievement highlights, but there are still some problems of the new curriculum reform. The author is mainly a summary of current situation of physical education teaching reform in recent years, and to study the problems in the process of reform, absorb excellent results, clearly erroneous zone existing in the teaching reform, and put forward the improvement scheme as makes every effort to provide a beneficial reference for PE teaching reform in colleges and universities.

\section{Conclusion}

In this paper, we conduct research on the development trend of modern sports teaching mode from the perspective of comprehensive physical ascension. The essence of the physical education teaching is generated, the default is a part of teaching students by nature; Teachers and students is the main part of the sports teaching activity, the physical education teaching activities generative includes generative practice activities, the generative process; Physical education teachers should not focus too much on the preset before class, but to daily teaching experience, for the real formation of teaching scene to provide the best help and support. In the future, we will combine more related theory to optimize our contemporary research result.

\section{References}

[1] Guo, Kaiqiang, and Juan Pu. "Researches of College Sports Technology Teaching Evaluation Methods." 2013 Conference on Education Technology and Management Science (ICETMS 2013). Atlantis Press, 2013.

[2] Fan, Hong. "Research on the Physical Education Teaching Effectiveness of the Higher Colleges and Universities with Intuitionistic Fuzzy Information." Journal of Convergence Information Technology 7.15 (2012).

[3] Liu, Li. "Research on the Status of Students' Participation in Sports in Some Private Middle Schools in Qingdao, China." Asian Social Science 10.13 (2014): 293.

[4] DU, Fang, et al. "" Constructivism" Joyful Sports Teaching Ideas of Interpretation." Sports Research and Education 2 (2013): 017. 
[5] LIU, Qiang, and Dong-fu XU. "Investigation and Study on the Present Situation of Sports Teaching in Elementary School of Wenshan Zhuang and Miao Autonomous Prefecture." Journal of Heze University 2 (2012): 036.

[6] Zhang, Yuan, and Wenge Yang. "Research on the Evaluation Index System of Sports Information of Colleges and Universities in Beijing." Organization 30 (2013): 70.
[7] Lin, Guo. "Experimental Study of Sports Training of Computer Supported Collaboration." Advanced Materials Research. Vol. 926. 2014.

[8] Lin, Z. H. U. "College Sports Curriculum Practice and Reform Countermeasures." Tribune of Education Culture 4 (2012): 026. 